light near the convergent to arrange itself in the form of an hyperbola, but no definiteness in the position of the axes could be detected other than a tendency for the visible hyperbola to occupy a quadrant opening toward the north or northeast. At many times during the hour the auroral display covered large sections of the southern sky, and the writer can remember thinking of the peculiar lateral shifting of the curtain in certain auroras and wondering how this would look if it took place near the convergent, but saw no such movement. At'times a shaft of light more or less meridional in direction lay across the convergent.

At the time the writer hoped that others were making similar observations and that it might be possible to determine the height of the point of convergence and he was somewhat surprised later to realize that his observations indicated the further fact of a change in the position of the convergent with reference to the stars which seemed only partly to be explained by their rotation. He only hopes that similar observations were made by others in different places and that the ones herein recorded are sufficiently accurate to make them of value. They at least have the merit of having been made by one who had no preconceived idea of what they might indicate, and who regrets, if they prove to have value, that he was unable to make use of more exact tools.

$$
\begin{aligned}
& \text { Lancaster D. Burling } \\
& \text { Geological Surtex, } \\
& \text { Ottawa, Canada }
\end{aligned}
$$

\section{THE DOMESTICATION OF THE LLAMA}

To the Editor of Science: A note in Science for March 15, 1918, by Mr. Philip Ainsworth Means, leads the reader to believe that the llama, alpaca, vicuna, and guanaco are distinct species and that the common belief is that all have been domesticated to some degree.

Prior to about 1890 there was great confusion regarding the specific status of these four animals, though the prevailing theory was that the llama had been derived from the guanaco and the alpaca from the vicuna. It is now known that the vicuña has never been domesticated, and that the alpaca and the llama are both domesticated forms of the wild guanaco. ${ }^{1}$ In view of the conspicuous differences between these two tame races of the guanaco it is easy to believe that a very long period of actual domestication has obtained, for the alpaca has been bred for his wool and the llama has been developed as a beast of burden as effectually as any of our races of domestic animals have been produced for special purposes by the most careful selective breeding.

The llama and the alpaca are not known in a wild state, though they of course occur, as do almost all other domesticated species, in a semi-wild or feral condition. They represent one of the rare cases of true domestication of an animal, and one of the still rarer cases where the ancestral species is known and still exists as a wild creature. Contrary to the statement in ScIENCE, they do breed freely in confinement; but since so many wild animals propagate regularly in captivity this can hardly be considered a test of true domestication. N. Hollister

$$
\begin{aligned}
& \text { National Zoological Park, } \\
& \text { Washington, D. C., }
\end{aligned}
$$

\section{THE AUDIBILITY OF SOUND}

RePLYING to the suggestion of Mr. Willard J. Fisher, in your issue of April 26, that an investigation be made of the area about Halifax with regard to audibility of the sound from the great explosion there, it may interest you to know that such an investigation was undertaken by the National Geographic Society not long after the occurrence of the explosion and that a quantity of data has been accumulated which is to be charted and tabulated as soon as other work will permit.

\section{Charles E. Munroe}

\section{SCIENTIFIC BOOKS}

The American Indian. An Introduction to the Anthropology of the New World. By Clark Wissler, Curator of Anthropology in the American Museum of Natural History, New York City. New York, 1917. Pp. xiii, 435 .

1 Thomas, Proc. Zool. Soc. London, 1891, pp. 885-387. 